\title{
Design of A Framework for Wireless Body Area Network Health Measurement Systems
}

\author{
Daniel Salcedo Pena \\ Electronic Systems's Postgraduate Program \\ Universidad Nacional Autónoma de México (UNAM) \\ Coyoacán, Mexico City, Mexico
}

\author{
Fatima Moumtadi, PhD \\ Engineering Faculty \\ Universidad Nacional Autónoma de México (UNAM) \\ Coyoacán, Mexico City, Mexico
}

\begin{abstract}
In this work we propose a design for embedded firmware to boost the development of Wireless Body Area Network systems for measuring of physiological data.
\end{abstract}

Keywords- WBAN, Embedded system, Physiological measurements.

\section{INTRODUCTION}

Wireless Body Area Network, WBAN is a standard for short-range, wireless communications in the vicinity of, or inside, a human body. It uses existing industrial scientific medical (ISM) bands as well as frequency bands approved by national medical and/or regulatory authorities [1]. Under this standard, a network of multiple miniature devices can be created to unobtrusively measure physiological data from an user.

Measuring and collecting reliable and consistent physiological data of a patient is a key part of health care. Activities such as diagnosis, after surgery monitoring or the control of chronical diseases rely on this data. Even sometimes a continuous every day measuring is required.

To this end, a large market of wearable devices was born, covering single variable devices to highly specialized multi variable systems. Single variable devices are the most versatile of all. They can be used regardless of the medical treatment as they are independent of data interpretation. On the other hand the specialized systems can integrate multiple sensors and data processing methods to present the user with a more useful interpretation of the data according with the medical context.

This paper proposes an open flexible firmware framework design for star-alike architecture WBAN systems development. This framework integrates a set of open libraries for data acquisition, data security, data processing and data transmission. Whereas the implementation of these functionalities has been based on a specific hardware design, the aim of this project is to be as hardware flexible as possible.

\section{WBAN ARCHITECTURE}

A Wireless Body Area Network (WBAN) system is a coordinated multiple nodes wireless network system. The two most common design shapes are Mesh and Star configurations. In a Mesh configuration every device works as a hub to extend the network far beyond the reach of a single transmitter, and so, each member of the network receives and retransmits the messages. A Star-alike architecture is a more simple design, but it requires two different types of members in the network: Concentrators and Peripherals.

The star architecture diagram is shown in figure 1. In this topology the system is composed by two classes of devices: a central module and its peripherals. The central module is responsible for setting up and driving the WBAN in any of the protocols defined in the standard: [1]

- IEEE 802.11 WLAN [2]

- IEEE 802.15.1 Bluetooth [3]

- IEEE 802.15.4 ZigBee [4]

- IEEE 802.15.4a Ultra Wide Band (UWB) ZigBee [5]

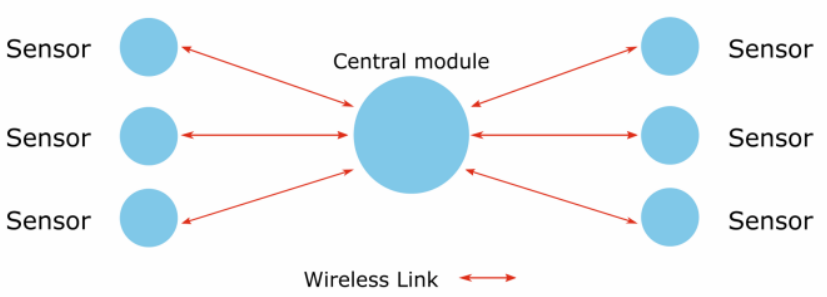

Fig. 1. Star architecture of a WBAN system.

\section{ARCHITECTURE MEMBERS TASKS}

In a Star Architecture, two different types of devices are needed. In figures $2 a$ and $2 b$ a general example of basic functionality for each type is shown.

Three function classes are required. The base class includes all the functions shared by the two types of devices, the concentrator class refers to the specific functions of the concentrator device and the peripheral class groups the exclusive functions of the peripheral devices. 


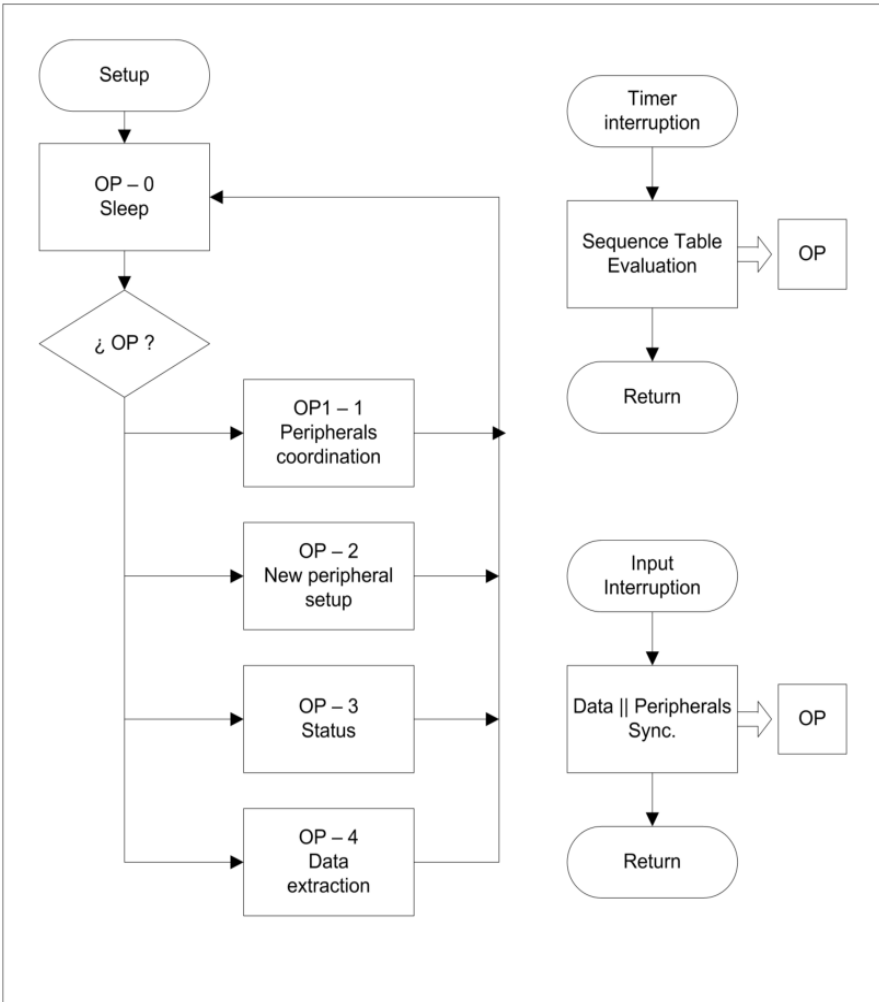

(a)

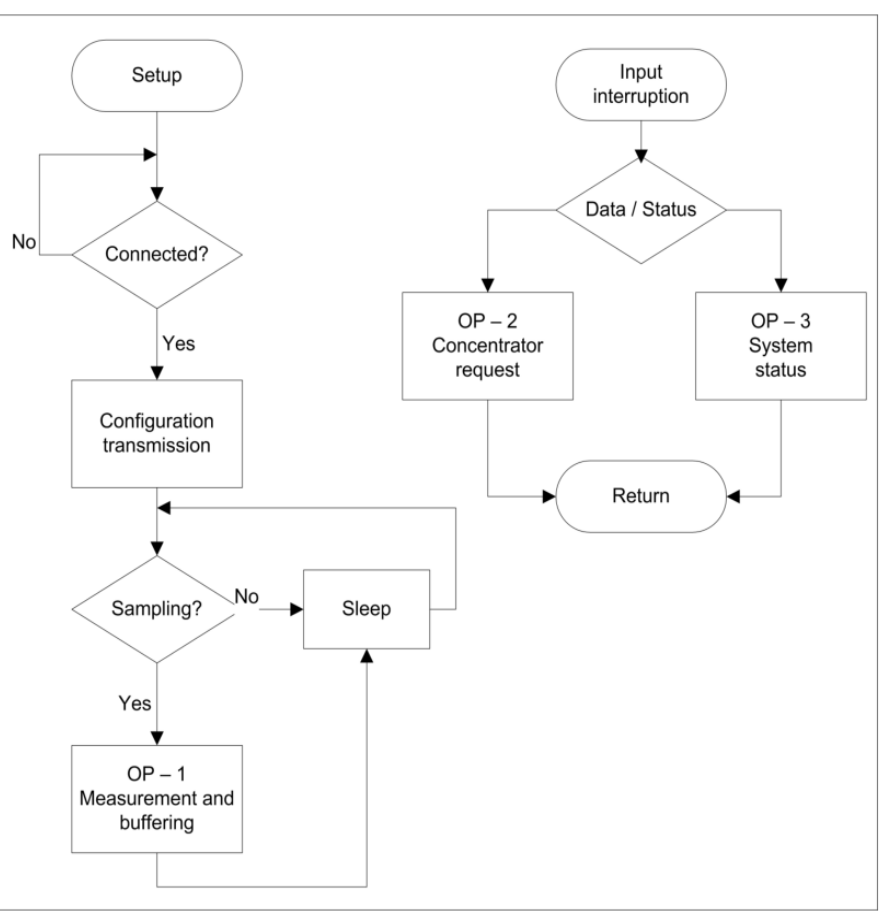

(b)

Fig. 2. Example algorithms for (a) concentrator and (b) peripheral devices.

\section{A. The base class}

The base class integrates the software core that runs a basic embedded system divided in categories:

- CPU / MCU setup

- Energy management

- Memory management

- Data transference

- Data security

\section{B. The concentrator class}

The main difference between a concentrator and a peripheral is that a concentrator doesn't deploy any physiological sensor. It is charged of network setup, data receiving and treatment, but most importantly, peripherals management. The functions required are organized in these categories:

- Peripherals management

- Data extraction

- Data synchronization

- Energy management

\section{The peripheral class}

The peripherals in this architecture handle the core instrumentation process. Multiple sensing technologies should be covered by this class, or at least be easily available to integrate

- Peripheral link

- Analog sensors library

- Digital sensors library

\section{THE IMPLEMENTATION}

This framework was first developed for a specific hardware configuration (based on a Microchip's 8 bit PIC microcontroller and an Arduino system) $[6,7]$ allowing us to test the basic functionalities and the integration of two different hardware systems (Figure 3)

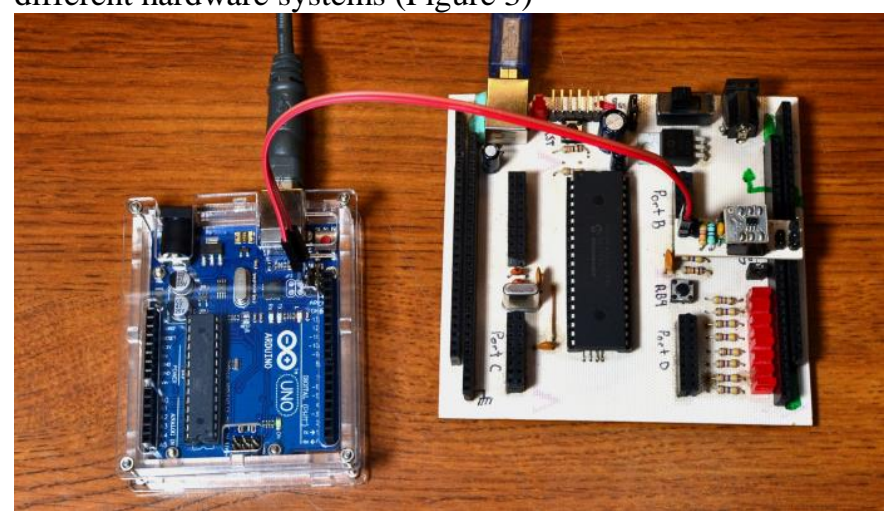

Fig. 3. Testing implementation.

\section{RESULTS}

Two sets of libraries have been developed following the design of this framework. One for a Microchip microcontroller and one for an Arduino system. As for now, limited capabilities are implemented as well as the number of sensors supported, but all of them fit on a broader firmware system. 


\section{CONCLUSION}

An open embedded framework for physiological WBAN measurement systems can help reduce the time of developing and testing of new prototypes, even if they rely on different technologies. To do so, this framework should be organized to fit the purpose of every module individually.

Further work is needed to add support for more variables and to include more technologies into this ecosystem.

\section{REFERENCES}

[1] IEEE Standard, "IEEE Standard for Local and metropolitan area networks - Part 15.6: Wireless Body Area Networks," IEEE Std 802.15.6-2012, pp. 1-271, Feb 2012.

[2] IEEE Standard, "IEEE Standard for Information technologyTelecommunications and information exchange between systems Local and metropolitan area networks-Specific requirements Part 11: Wireless LAN Medium Access Control (MAC) and Physical Layer (PHY) Specifications," IEEE Std 802.11-2012 (Revision of IEEE Std 802.11-2007), pp. 1-2793, March 2012.

[3] IEEE Standard, "IEEE Standard for Information technology- Local and metropolitan area networks- Specific requirements- Part 15.1a:
Wireless Medium Access Control (MAC) and Physical Layer (PHY) specifications for Wireless Personal Area Networks (WPAN)," IEEE Std 802.15.1-2005 (Revision of IEEE Std 802.15.1-2002), pp. 1-700, June 2005.

[4] IEEE Standard, "IEEE Standard for Local and metropolitan area networks-Part 15.4: Low-Rate Wireless Personal Area Networks (LRWPANs)," IEEE Std 802.15.4-2011 (Revision of IEEE Std 802.15.42006), pp. 1-314, Sept 2011

[5] IEEE Standard, "IEEE Standard for Information Technology Telecommunications and Information Exchange Between Systems Local and Metropolitan Area Networks - Specific Requirement Part 15.4: Wireless Medium Access Control (MAC) and Physical Layer (PHY) Specifications for Low-Rate Wireless Personal Area Networks (WPANs)," IEEE Std 802.15.4a-2007 (Amendment to IEEE Std 802.15.4-2006), pp. 1-203, 2007.

[6] Microchip Technology Inc. "8-bit pic and avr mcus". Available at: http://www.microchip.com/design-centers/8-bit, (Accessed on July 26, 2018)

[7] Arduino AG. "What is arduino?" Available at: https://www.arduino.cc/en/guide/introduction (Accessed on July 26, 2018) 